\title{
Generieren lastgerechter Materialparameter für FEM-gestützte Umformprognosen - am Beispiel von Karton-Verbundmaterialien
}

Toma Schneider, Antje Harling, Frank Miletzky

Zwei wesentliche Vorrausetzungen zum Aufbau mechanischer Verhaltensprognosen auf Basis der finiten-Element-Methode (FEM) sind die Verfügbarkeit von Materialmodellen sowie zugehörige Messverfahren zur Parameterbestimmung. Gegenstand dieser Abhandlung ist die Vorstellung einer neuartigen Messmethodik zur Erhebung des plastischen Biegeund Faltverhaltens von faserbasierten Verbundmaterialien und dessen Anwendung zum vereinfachten Aufbau von numerischen Struktursimulationen. Als besonderes Merkmal sei dabei der Ansatz einer äußeren, integralen Verhaltensbeschreibung der vielfältigen Vorgänge auf der Mikrostrukturebene des Materials genannt. Damit wird es möglich das mechanische Strukturverhalten komplexer Makrostrukturen, wie komplette Verpackungen auf Basis von Karton-Verbundmaterial, zu untersuchen und damit Optimierungen hinsichtlich Versagensverhalten und Materialeffizienz durchzuführen.

Schlagworte: plastisches Umformen, Biegen, Falten, Materialverhalten, Prozessprognose, FEM-Simulation.

\section{Einleitung}

Der Einsatz von Karton-Verbundmaterialien in der Verpackungsproduktion ist eine Möglichkeit zur Realisierung eines vorteilhaften Distributions- und Gebrauchsverhaltens von Konsumgütern. Der mehrlagig aufgebaute und zumeist beschichtete Karton erfüllt dabei unterschiedliche Aufgabenstellungen, zur Realisierung unterschiedlicher Verpackungsfunktionen. Als wesentliche Funktionen seien hier die Schutz-, Lager-, Transport- und Kommunikationsfunktion genannt. Bei der Realisierung dieser Funktionen treten unterschiedliche Problemlagen und Zielkonflikte auf. Die Verpackung soll maximalen Schutz bei möglichst wenig Materialeinsatz bieten, der Verarbeitungsprozess muss materialschonend, stabil und schnell zu gestalten sein. Dabei sollte die Umsetzung in der Verarbeitungsmaschine wenig Bauraum und Energie verbrauchen sowie geringe Emissionen verursachen. Die Gestaltung und Optimierung derartig komplexer Makrostrukturen und zugehöriger Verarbeitungsprozesse kann durch Einsatz numerischer Prognosewerkzeuge unterstützt werden. 
Der im Maschinenbau seit Jahren breite und etablierte Einsatz von FEM-Werkzeugen führt in der Papiertechnik zur Prozessprognose von Verarbeitungsvorgängen aktuell nur ein Nischendasein. Als eine Ursache kann die unzureichende Verfügbarkeit von Beschreibungsmodellen des Materialverhaltens identifiziert werden, insbesondere im Bereich plastischer Verformung. Zum Aufbau von Makrostrukturmodellen ist eine Materialbeschreibung mindestens auf Mesostrukturebene erforderlich. Diese soll nun mittels neuartiger Messmethodik entscheidend ausgebaut werden. Die damit einhergehende höhere Verfügbarkeit von einsatzbereiten Prognosewerkzeugen hilft neue Anwendungsbereiche zu erschließen, Entwicklungsprozesse signifikant zu verkürzen und ermöglicht so zugleich einen Beitrag zur Ressourcenschonung durch Realisierung hoher Materialeffizienz.

\section{Umformprozesse}

Formgebende Prozesse bei der Herstellung von Verpackungen aus flächigen Halbzeugen werden zumeist durch Biege- und Faltumformung sowie Tiefziehen realisiert. Das Ziel der Bestrebungen ist die Bereitstellung lastgerechter Materialparameter zur Beschreibung von plastisch, eindimensional verformten Strukturen aus Verbundmaterial durch Zug-, Druck- und Biegebeanspruchung. Mehrdimensionale, große plastische Verformungen durch Beulen, wie beim Tiefziehen, sind nicht Gegenstand der Untersuchungen.

\section{Biegen}

Beim einachsigen Biegen (vgl. Abbildung 1 li.) durch Querkraft- oder Drehmomenteinwirkung wirkt eine kombinierte Zug- und Druckbeanspruchung im belasteten Querschnitt. Dabei liegt eine lineare Dehnungsverteilung in Dickenrichtung des Materialverbundes vor, der Nulldurchgang von Dehnung und Spannung wird als neutrale Linie bezeichnet (siehe Abbildung 6 re.).

\section{Falten}

Das Falten (vgl. Abbildung 1 re.) wird durch einen vorgelagerten Biegebruch und die Ausbildung mehrerer Biegequerschnitte charakterisiert. Der Biegebruch erfolgt beim Überschreiten von inneren Materialfestigkeiten. Es gibt zwei wesentliche Versagensarten: Den Zugbruch des äußersten Bereiches, typisch für homogene Materialien oder den Schubbruch in flächiger Ausbreitungsrichtung bei heterogenen Materialien oder Laminaten. Der Schubbruch teilt den Lastquerschnitt in zwei unabhängige Biegequer- 
schnitte und bewirkt eine lokale Delamination des Verbundes. Durch räumliches Ausweichen der Teilquerschnitte voneinander bildet sich ein Faltgelenk aus, welches kennzeichnend für das Falten ist.

Biegen:

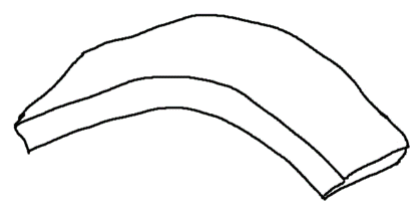

- plastische Dehnungen

- Fließen infolge von Mikrostrukturbrüchen

- keine Delamination durch Mesostrukturbrüche

- nur relativ große Kantenradien erzielbar
Falten:

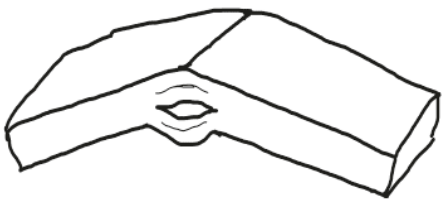

- Delamination durch Mesostrukturbrüche

- (Schub und Zug)

- Querschnittsauflösung per Materialverschiebungen

- Faltgelenkausbildung (Biegeumformung der Teilquerschnitte)

- geringe Kantenradien realisierbar

Abbildung 1: Umformen mehrlagiger Faserverbundwerkstoffe:

Biegen und Falten (Schmidt \& Schneider, 2006)

\section{Materialbeanspruchung und Messmethodik}

Ein Merkmal von faserbasierten, bahnförmig und kontinuierlich produzierten Materialien, wie Papier und Karton ist die Ausrichtung der Fasern auf Mikrostrukturebene. Dies äußert sich in einem richtungsabhängigen mechanischen Materialverhalten. Zur Beschreibung der elastischen Anisotropie nur einer Materiallage sind bereits neun unabhängige Materialparameter erforderlich (Hahn, 1992). Eine induktive Methode zur Bestimmung des integralen Verbundverhaltens scheitert unter anderem an dem Parameterumfang, dem Mangel geeigneter Materialprüfverfahren und der Komplexität des erforderlichen Mikrostrukturmodells. Deshalb wird ein Ansatz der integralen Beschreibung von außen gewählt. Dazu wird die äußere Lastreaktion auf ein vereinfachtes inneres Ersatzmaterialmodell abgebildet. Ohne Betrachtung des Beulens kann das Materialmodell auf die Abbildung der Hauptbeanspruchungsrichtung reduziert werden. Dies ist für eine Modellierung realer Biege- und Faltvorgänge ausreichend. Da 
papierfaserbasierte Materialien eine relative geringe Bruchdehnung besitzen, werden sie in der Regel auch nur für lokal einfach gekrümmten Umformungen eingesetzt.

\section{Zugbeanspruchung}

Für die Zugbeanspruchung kann dabei, wie bei homogenen Werkstoffen verfahren werden, indem eine Kraft-Weg-Kurve aus der Zugprüfung (DIN EN ISO 1924-2) in ein Spannungs-Dehnungs-Diagramm überführt wird. Die Spannung-Dehnungs-Kurve entspricht dabei der Summe der einzelnen Kurven der Materiallagen nach Multiplikation mit ihrem Schichtdickenanteil.

\section{Biegebeanspruchung und Falten}

Karton besitzt bereits im elastischen Bereich ein unterschiedliches Materialverhalten unter Zug- und Druckbeanspruchung (siehe Anfangsanstiege in Abbildung 5). Daraus folgt eine unsymmetrische Dehnungsverteilung bei der kombinierten Biegebeanspruchung. Für Faserwerkstoffe aus nur einer oder mehreren Lagen gleichen mechanischen Materialverhaltens kann das Verhalten unter Biegung, auf Basis des Längskraftgleichgewichtes, berechnet werden. Für typische, mehrlagig aufgebaute Kartone und Verbunde gelingt dies in Ermangelung des mechanischen Verhaltens der einzelnen Lagen und Ihrer Verbindungen nicht. Deshalb wird eine experimentelle Ermittlung des plastischen Materialverhaltens unter Biegebeanspruchung vorgeschlagen.

Naturfaserstoffe besitzen eine heterogene Materialverteilung auf Mikrostruktur- und teils Mesostrukturebene. Äußere Lasteinwirkungen können Lastgradienten verursachen. Daraus folgt, dass die Bestimmung lokaler Beanspruchungen häufig nicht möglich ist. Etablierte Verfahren und Anordnungen zur Biegesteifigkeitsmessung (Zweipunkt- und Dreipunktbiegung, DIN 53121) erfassen eine kombinierte Biege- und Schubverformung oder sind auf den elastischen Bereich begrenzt (Vierpunktbiegung, DIN 53121). Durch unterschiedliche Experimente wurde nachgewiesen, dass der Schubanteil der Verformung bei der üblichen Zweipunktbiegung von Karton im zweistelligen Prozentbereich liegen kann. Der bei Prüfung unter Querkraftbiegung erhobene Verformungsweg ist die Summe aus Biege- und Schubverformung (siehe Abbildung 5). Eine nachfolgende Zuordnung der Verformungsanteile und die Ermittlung des reinen Biegeanteils im Bereich plastischer Verformung sind nur unscharf möglich.

Unter den vorgenannten Aspekten wurde zur Erfassung des Biegeverhaltens eine neuartige Probenanordnung nach dem Ringprinzip ausgewählt (siehe Abbildung 2). Dazu wird aus einer streifenförmigen Probe ein Ring geformt. Am fixierten Ende der Probe werden die Lagerkräfte und -momente in der Einspannung erfasst. Das lose Ende wird durch einen Führungsspalt nahe der Einspannung und einen Abzug geführt. Während 
mittels des Abzuges der Durchmesser des Ringes reduziert wird und damit die Krümmung steigt, steigt auch das Biegemoment in der Einspannung bis zum Biegebruch an. Die zu Beginn in Streifenrichtung nahezu konstante Krümmung entspricht der Division des Vollwinkels des Ringes durch seinen Umfang.

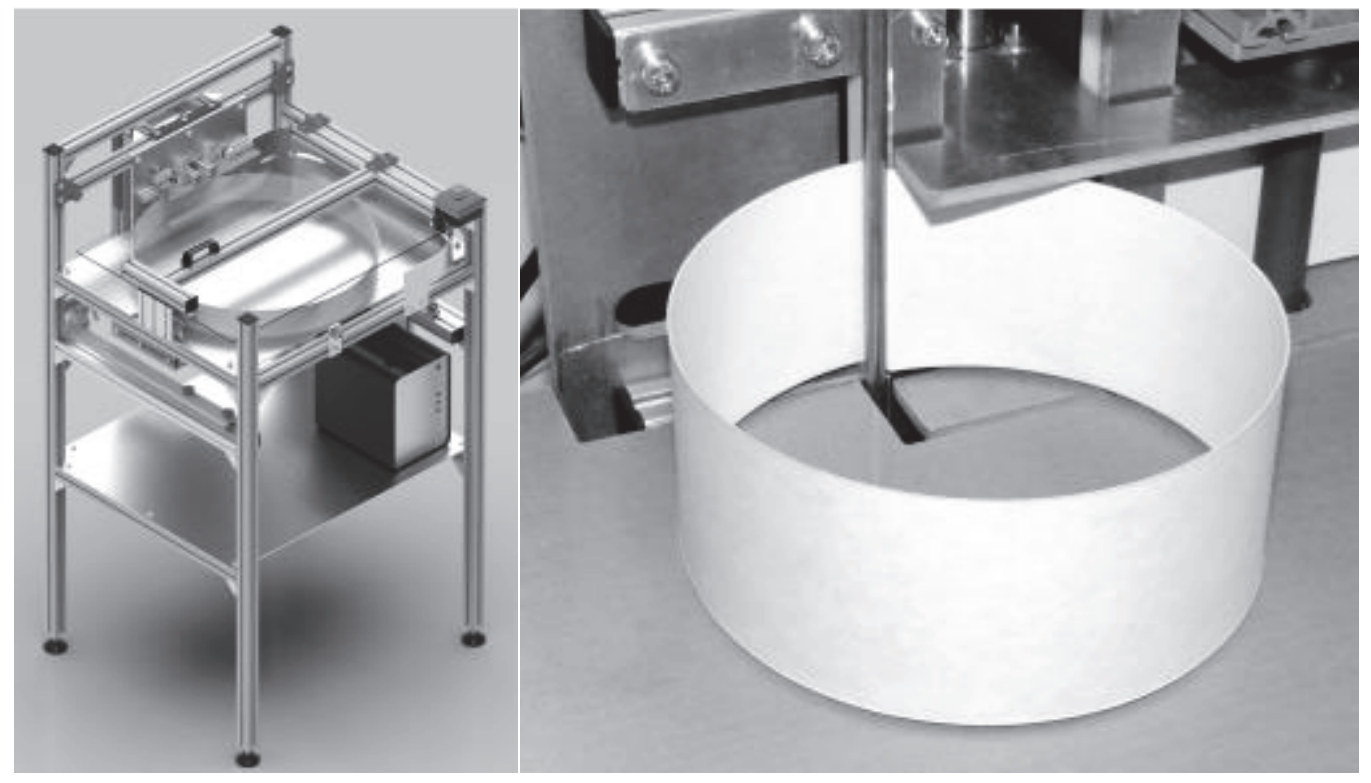

Abbildung 2: Prüfgerät Krümmungswiderstand an der Papiertechnischen Stiftung (PTS)

Mit dieser Anordnung kann die nichtlineare Übertragungsfunktion von Drehmoment zu Krümmung, kurz Krümmungswiderstand, direkt erhoben werden. Bei dem Absinken des Ringdurchmessers steigt der Fehlereinfluss des bauraumbedingten Versatzes der Einspannungen an und es bildet sich ein Spiralsegment aus. Des Weiteren verändert sich der anfangs fast konstante Krümmungsverlauf durch lokales Materialversagen beim Biegebruch. Mittels iterativer und inverser Methoden werden diese geometrischen, systematischen Fehler herausgerechnet. Dazu wird die sich kontinuierlich entwickelnde Übertragungsfunktion (siehe Abbildung 3 li.) perspektivisch interpoliert und per segmentiertem Geometriemodell auf Basis der Euler-Bernoulli-Balken-Theorie überprüft. Mit Hilfe von Referenzkurven ohne Biegestellenvorbereitung kann mittels dieses Verfahrens auch die Übertragungsfunktion von lokal vorhandenen Faltlinienvorbereitungen, wie dem Rillen, bestimmt werden (siehe Abbildung 3 re.). 


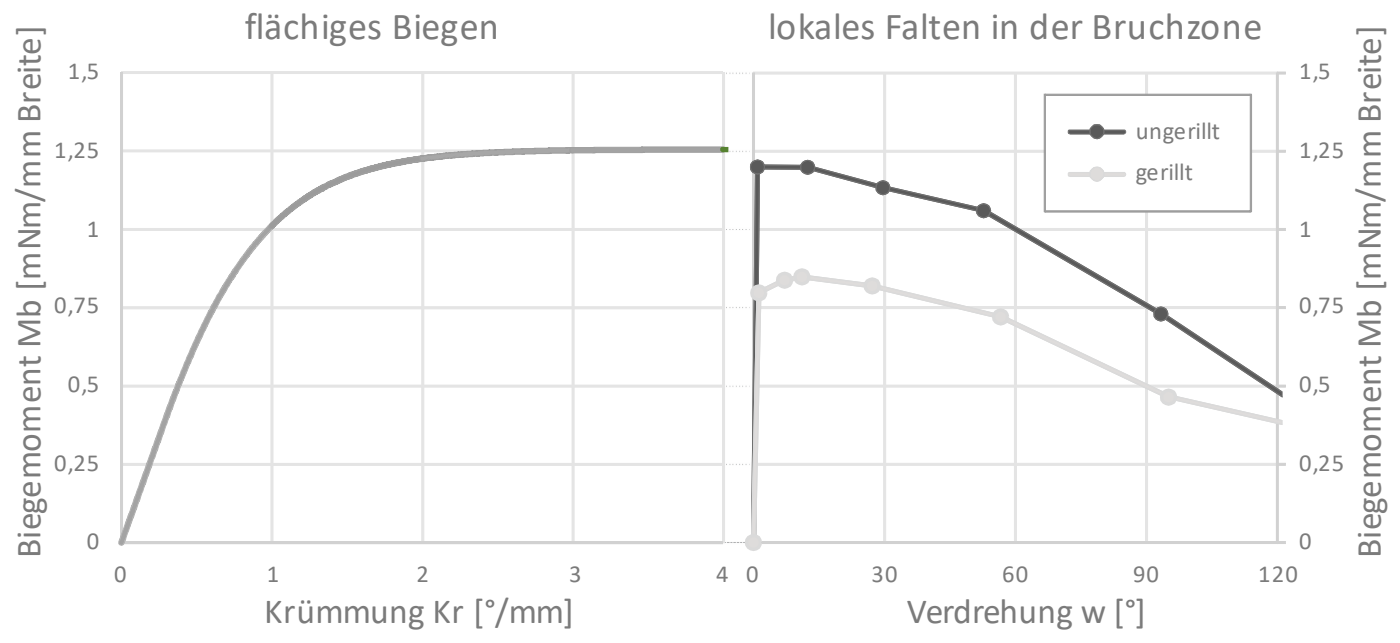

Abbildung 3: Übertragungsfunktionen für Biegen und Falten

\section{Druckbeanspruchung}

Die Drucksteifigkeit kann mit der Universalprüfmaschine oder mittels eines modifizierten Prüfgerätes zur Messung des Streifenstauchwiderstandes (SCT, in Anlehnung an DIN 54518) bei einer Probenlänge von 5 mm gemessen werden. Bei der Bereitstellung von Drucksteifigkeitskurven sind zwei wesentliche Probleme zu nennen: Als erstes neigen streifenförmige Proben unter Drucklast zum seitliche Ausknicken. Zur Erfassung plastischen Druckverhaltens wird daher ein sehr geringer Schlankheitsgrad (Länge/Dicke) der Probe gewählt. Die damit verbundene kurz gewählte Einspannlänge verstärkt das zweite Problem. Während der Messung gleitet ein Teil der Probe zwischen die Klemmbacken, dadurch wird ein zu großer Stauchungsweg und in Folge eine betragsmäßig zu große Dehnung ermittelt. Durch eine entsprechende Anpassung von Klemmbackendruck und Backenoberfläche kann dieses Problem reduziert, aber nicht beseitigt werden. Die gemessene Referenzkurve per modifiziertem SCT Gerät wurde mit Hilfe der Optischen Dehnfeldanalyse (ODA) korrigiert, wobei die Messunsicherheit noch steigt.

Eine alternative Möglichkeit zur Bereitstellung plastischer Druckspannungs-Dehnungsverläufe besteht in der Berechnung auf Basis von Zugversuch und Krümmungswiderstandsmessung. Dazu wurde ein Verfahren für Materialien mit homogener Mesostruktur entwickelt und überprüft (vgl. Abbildung 4). Die Berechnung der 
Druckspannungskurve erfolgt durch diskrete, mehrfach verschachtelte, iterative Näherungsberechnungen mittels Abgleich resultierender Längskräfte und Drehmomente bei vorgegebenen Grenzdehnungen und zugehörigen Spannungen.

Eine Nutzung dieses Vorgehens für mehrlagige Kartone und Verbunde gelingt unter der Annahme, dass das Zug- und Druckspannungsverhalten der einzelnen Kartonlagen mit jeweils einer Master-Materialkurve für Zug- und Druckbeanspruchung sowie zwei Proportionalitätsfaktoren zur Skalierung in Dehnungs- und Spannungsrichtung beschrieben werden kann. Das zusätzlich erforderliche Materialverhalten der Beschichtungen kann aus Materialdatenbanken entnommen werden. Eine weitere Vereinfachung besteht in der Verwendung gleicher Proportionalitätsfaktoren für Zug und Druck. Dies bedeutet z. B., wenn die Kartonmittelage dreimal zugweicher als die Kartondecklage ist, so ist sie auch dreimal druckweicher als die Decklage. Da Karton und Verbundmaterialien meist unsymmetrisch aufgebaute Biegebalken darstellen, gibt es durch Faltung unter Einschluss der Außen- oder Innenseite zwei unterschiedliche plastische Krümmungswiderstandskurven für denselben Lastquerschnitt. Somit muss für beide Faltrichtungen bei richtig gewählten Proportionalitätsfaktoren im Ergebnis die gleiche Drucksteifigkeitskurve berechnet werden. Unter diesen Voraussetzungen können iterativ die Proportionalitätsfaktoren und die Drucksteifigkeitskurve des gesamten Verbundes bestimmt werden. Eine erste Umsetzung in Form eines Berechnungsprogramms für mehrlagige Kartone und Verbunde ist bereits erfolgt, die umfassende Validierung und Überprüfung der getroffenen Annahmen steht noch aus.

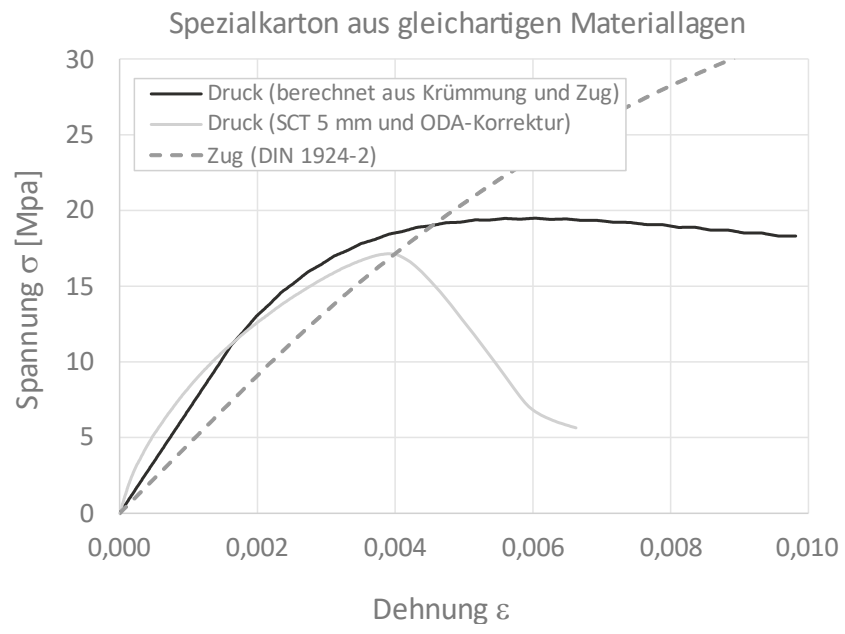

Abbildung 4: Vergleich ermittelter Druckspannungs-Kurven (Beträge) 


\section{Schubbeanspruchung}

Es gibt unterschiedliche Verfahren zur Ermittlung von Schub-Eigenschaften. Für faserverstärke Kunststoffe und Intra-laminarem Schub (in Plane) z.B. die DIN EN ISO 14129 und für Inter-laminarem Schub (out of Plane) z.B. die DIN EN ISO 14130.

An der Papiertechnischen Stiftung (PTS) ist eine Vorrichtung zur out of Plane-Messung vorhanden (Matheas, 2015). Die Probenvorbereitung für dieses Prüfverfahren ist sehr aufwändig, daher wird eine indirekte Erhebung zur Bereitstellung von Schubsteifigkeiten vorgeschlagen. Für den elastischen Bereich kann dies durch Kombination von Zweipunktbiegung und Krümmungswiderstandsmessung auf Basis der Differenzialgleichung der Biegelinie nach der Timoschenko-Balkentheorie (Öchsner, 2016) erfolgen. Dazu wird mittels Krümmungswiderstandsmessung der Biegeanteil bei der Zweipunktbiegung bestimmt, durch Subtraktion von der Gesamtverformung kann die Schubverformung und Schubsteifigkeit berechnet werden. In Abbildung 5 sind diese Anteile für einen typischen Faltschachtelkarton dargestellt.

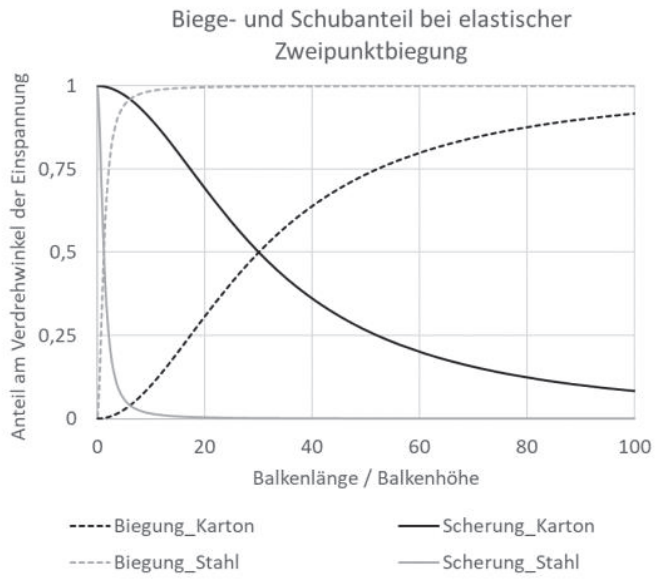

Abbildung 5: Verformungsanteile in Abhängigkeit der Schlankheit des Biegebalkens

\section{Parameterbereitstellung für FEM-Anwendungen am Beispiel der Software ANSYS}

Zur Abbildung von Makrostrukturen auf Basis flächiger Werkstoffe hat sich eine Modellierung der Geometrie aus Schalenelementen bewährt. Diese Elemente können ein- 
oder mehrlagig abgebaut werden, wobei das geometrische Merkmal Dicke über Parameter zugewiesen werden kann. Diese Eigenschaft ist eine wesentliche Voraussetzung für das hier aufgezeigte Vorgehen der belastungsgerechten Materialmodellierung. Es besteht die Vorauswahl einer elastischen, anisotropen oder einer plastischen, isotropen Beschreibung des Materialverhaltens mit Proportionalitätsfaktoren für anisotropes Fließverhalten. Nachfolgend wird vorrangig auf das plastische Verhalten eingegangen, welches über einen multilinearen Spannungs-Dehnungs-Verlauf beschrieben wird. Zur Abbildung von plastischen Zug- und Druckbeanspruchungen kann somit jeweils eine diskretisierte Spannungs-Dehnungs-Kurve aus den Materialprüfungen genutzt werden.

Die Abbildung der Biegebeanspruchung erfolgt über ein berechnetes Pseudo-Materialverhalten. Dazu wird auf Basis des gemessenen Biegemoment-Krümmungs-Verlaufes mit Hilfe der Formeln (1) und (2) und der vorzugebenen Gesamtschichtdicke die zugehörige Spannungs-Dehnungs-Kurve invers berechnet (siehe Abbildung 6). Dieses Prinzip gibt die Elementverformung unter äußerer Lasteinwirkung gut wieder und erfordert keine Modellabbildung der inneren Strukturänderungen beim plastischen Biegen. Bei der Auswertung von Simulationsergebnissen ist jedoch darauf zu achten, dass nur Reaktionskräfte und -momente sowie Verformungen und Dehnungen direkt ausgewertet werden können. Die berechneten Spannungen sind in der Regel prinzipbedingt zu gering, da eine vereinfachte homogene Steifigkeitsverteilung zu Grunde gelegt wird.
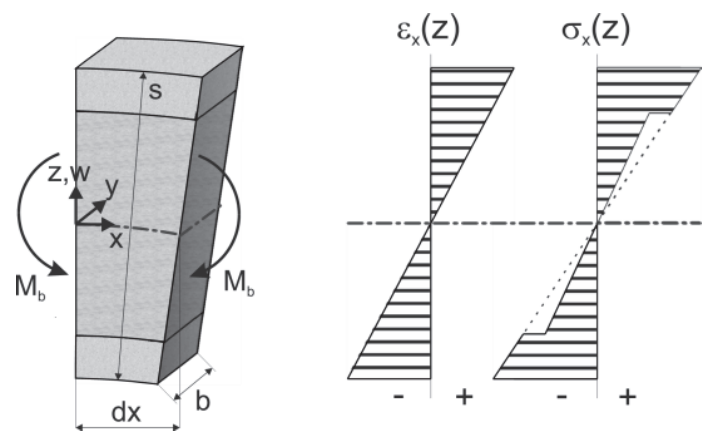

$$
\begin{aligned}
& M_{b}=\text { Biegemoment } \\
& \sigma_{x}=\text { Normalspannung (x-Rich- } \\
& \text { tung) } \\
& \mathrm{Kr}=\text { Krümmung (um y-Achse) } \\
& \varepsilon_{\mathrm{x}}=\text { Dehnung (x-Richtung) } \\
& \mathrm{s}=\text { Materialdicke (z-Richtung) } \\
& \mathrm{b}=\text { Breite (y-Richtung) }
\end{aligned}
$$

$$
\begin{aligned}
& K r(x)=\arctan \left(\frac{2 * \varepsilon_{\mathrm{x}_{\max }}(x)}{s}\right) \approx\left(\frac{2 * \varepsilon_{\mathrm{x}_{\max }}(x)}{s}\right), \\
& M_{b}(x)=b * \int_{0}^{s} z * \sigma_{x}(z) d z .
\end{aligned}
$$

Abbildung 6: Spannungsverteilung über dem Querschnitt beim Biegen (Schmidt \& Schneider, 2006) sowie Berechnung von Krümmung und Biegemoment auf Basis der Spannungs-Dehnungs-Kurve 
Eine Abbildung von lokalen Rill- oder Faltlinien erfolgt im Strukturmodell durch diskrete Rotationsfedern, als Koppelemente des Verdrehungsfreiheitsgrades zwischen zwei benachbarten Schalenelementen. Die Parametrisierung in Form eines grobdiskreten Drehmoment-Winkel-Verlaufes basiert auf den Werten aus der Krümmungswiderstandsmessung (Abbildung 3, re.).

\section{Experimentell-numerischer Modellabgleich}

Mittels numerischer Modelle der Prüfverfahren wurden die Material- und Strukturmodelle experimentell-numerisch verifiziert (siehe Abbildung 7). Die prognostizierten Verläufe der Arbeitsdiagramme bilden die experimentell ermittelten Verläufe ab. Einzig die Zweipunktbiegung mit kurzer Biegebalkenlänge wird zu steif wiedergegeben. Da bisher keine Lösung zur Implementierung der wesentlich geringen relativen Schubsteifigkeit von Karton in Verbindung mit plastischem Biegen gefunden wurde, können als Abhilfe, insbesondere bei einer Umformung quer zur Faserrichtung, die Bereiche unter hoher Querkraft- aber geringer Drehmomentbeanspruchung mit einem elastischen, schubweichen Materialverhalten abgebildet werden.

In einem zweiten Schritt folgt eine Modellverifizierung anhand eines komplexen Makrostrukturmodells unter äußerer Lasteinwirkung. Dazu ist eine mehrschrittige Berechnung und Modellanpassung vorgesehen. Dabei wird das Materialverhalten der einzelnen finiten Elemente entsprechend ihrer ermittelten Hauptbeanspruchung zyklisch angepasst. Es sollen Algorithmen zur Automatisierung der Vorauswahl des Materialmodells entwickelt und erprobt werden.

parametrisches ANSYS ${ }^{\circledR}$-Strukturmodell aus Schalenelementen (flächiges Biegen) und Rotationsfedern (lokales Falten)

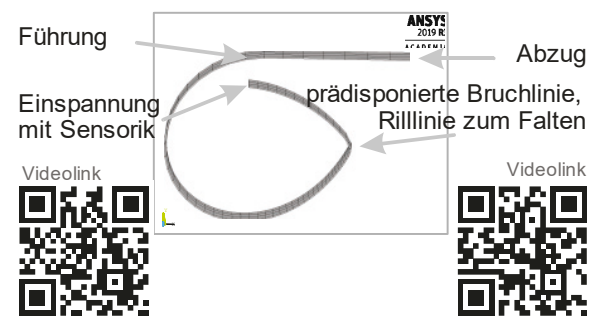

Krümmungswiderstand - Faltung cd

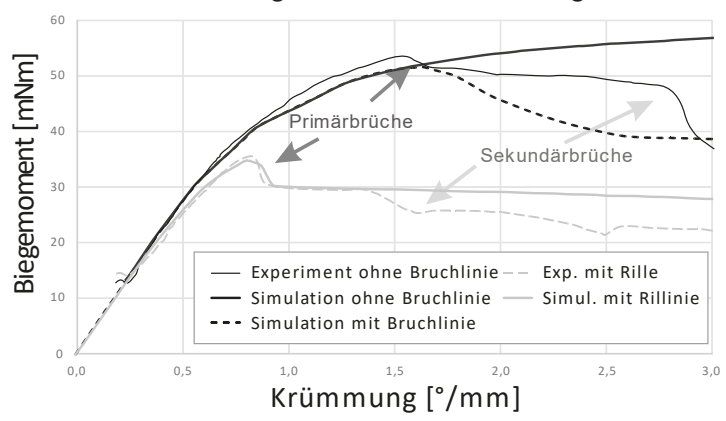

Abbildung 7: Strukturmodell Ringversuch sowie experimentell-numerischer Abgleich der Krümmungswiderstands-Messung per Arbeitsdiagramm 
Bei homogenen Materialien und rechteckigem Lastquerschnitt ist die Widerstandskraft gegen Zug und Druck proportional zur Querschnittshöhe bzw. Gesamtdicke des Material. Bei gerader Biegung liegt eine kubische Proportionalität zwischen Widerstandsmoment und Gesamtdicke vor. Eine identische äußere Lastreaktion kann mit unterschiedlichen Dicken und Steifigkeiten realisiert werden. Durch gezielte Abstimmung der Parameter Schichtdicke sowie Zug- und Biege- oder Druck- und Biegesteifigkeit kann ein kombiniertes Ersatzmaterialverhalten bereitgestellt werden, welches im elastischen Bereich jeweils zwei Lastfälle abbildet und im plastischen Bereich priorisiert werden kann.

\section{Zusammenfassung und Ausblick}

Auf Basis der neuartigen Messmethodik Krümmungswiderstand können plastisches Biegen und Falten von mehrlagigen Faserverbundwerkstoffen parametrisch beschrieben und für numerische Makrostruktursimulation bereitgestellt werden. Die dazu geschaffenen Programmabläufe konvertieren die Messdaten in Ersatzmaterialverhalten und stellen diese als Input-files für FEM-Anwendungen zur Verfügung. Die damit erheblich abgesenkte Eintrittsschwelle zur Entwicklung einfacher Umformprognosen, erleichtert insbesondere kleinen und mittelständische Unternehmen der Verarbeitungsbranche die ökonomische Nutzung von FEM-Werkzeugen.

Unabhängig von der Bereitstellung von Materialparametern für FEM-Anwendungen können mittels der Krümmungswiderstandsmessung Materialien hinsichtlich ihres Biege- und Faltverhaltens erstmals unabhängig von der Probengeometrie charakterisiert werden. Dazu wird die Biegemoment-Krümmungs-Kurve auf das maximale Biegemoment normiert. Der Anstieg und die Geometrie der Kurve gibt Auskunft über die Art des zu erwartenden Versagens beim plastischen Biegen zwischen den Grenzen weiträumigen Knautschens und lokalen Brechens. Materialien mit identischem normierten Krümmungsverlauf verhalten sich, trotzt unterschiedlicher Biegesteifigkeit, bei weggesteuerten Umformprozessen vergleichbar. Dies ist besonders bei Umstellung bestehender Prozesse auf neue Halbzeuge und der Entwicklung alternativer Biegestellenvorbereitungen von großem Nutzen. Des Weiteren zeigte sich in Vorversuchen, dass die normierte Kurve des Krümmungswiderstands als Indikator für erfolgreiches Rillen und Tiefziehen dienen kann.

Das Projekt IK-VF 190003 „Krümmungswiderstandsmessung“, Laufzeit 06/201911/2021, wird aus Mitteln des Bundeswirtschaftsministeriums für Wirtschaft und Energie (BMWi) gefördert. 


\section{Literaturverzeichnis}

Groth, C., \& Müller, G. (2002). FEM für Praktiker - Grundlagen. Bd. Band 1. Renningen: expert Verlag.

Hahn, H. G. (1992). Technische Mechanik fester Körper. München Wien: Carl Hanser Verlag.

Matheas, J. (2015). Materialparameter für effiziente Beurteilung der mechanischen Eigenschaften von Mehrschichtmaterialien und den darauf aufbauenden Entwurf angepasster Produkte. PTS-Forschungsbericht VF120017. Heidenau: Papiertechnische Stiftung.

Öchsner, A. (2016). Theorie der Balkenbiegung. Southport, QLD, Australien: Springer Vieweg.

Schmidt, F. J., \& Schneider, T. (2006). Anwendung der Impact-Verarbeitung bei der Umformung von Kartonverbundwerkstoffen. Tagungsband WD 2006. Dresden: Selbstverlag der TU-Dresden.

\section{Kontakt}

Dipl.-Ing. Toma Schneider, Dr. Antje Harling

Papiertechnische Stiftung (PTS)

Pirnaer Straße 37

D-01809 Heidenau

www.pts-paper.de

Prof. Dr. Frank Miletzky

Technische Universität Dresden

Institut für Naturstofftechnik

01307 Dresden

www.tu-dresden.de

Gefördert durch:

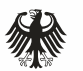

Bundesministerium für Wirtschaft und Energie

aufgrund eines Beschlusses

des Deutschen Bundestages 